\title{
The number of small covers over cubes
}

\author{
SUYOUNG ChOI
}

\begin{abstract}
In the present paper we find a bijection between the set of small covers over an $n$-cube and the set of acyclic digraphs with $n$ labeled nodes. Using this, we give formulas of the number of small covers over an $n$-cube (generally, a product of simplices) up to Davis-Januszkiewicz equivalence classes and $\mathbb{Z}_{2}^{n}$-equivariant homeomorphism classes. Moreover we prove that the number of acyclic digraphs with $n$ unlabeled nodes is an upper bound of the number of small covers over an $n$-cube up to homeomorphism.
\end{abstract}

37F20, 57S10; 57N99

\section{Introduction}

Let $P$ be a simple convex polytope of dimension $n$ and let $\mathcal{F}(P)=\left\{F_{1}, \ldots, F_{m}\right\}$ be the set of facets of $P$. Consider $\lambda: \mathcal{F}(P) \rightarrow \mathbb{Z}_{2}^{n}$ which satisfies the nonsingularity condition: $\left\{\lambda\left(F_{i_{1}}\right), \ldots, \lambda\left(F_{i_{n}}\right)\right\}$ is a basis of $\mathbb{Z}_{2}^{n}$ whenever the intersection $F_{i_{1}} \cap \cdots \cap F_{i_{n}}$ is nonempty. We call $\lambda$ a characteristic function. Let $\mathbb{Z}_{2}\left(F_{i}\right)$ be the subgroup of $\mathbb{Z}_{2}^{n}$ generated by $\lambda\left(F_{i}\right)$.

Given a point $p \in P$, we denote by $G(p)$ the minimal face containing $p$ in its relative interior. Assume $G(p)=F_{j_{1}} \cap \cdots \cap F_{j_{k}}$. Then $\mathbb{Z}_{2}(G(p))=\bigoplus_{i=1}^{k} \mathbb{Z}_{2}\left(F_{j_{i}}\right)$. Note that $\mathbb{Z}_{2}(G(p))$ is a $k$-dimensional subgroup of $\mathbb{Z}_{2}^{n}$. Let $M(\lambda)$ denote $P \times\left(\mathbb{Z}_{2}\right)^{n} / \sim$, where $(p, g) \sim(q, h)$ if $p=q$ and $g^{-1} h \in \mathbb{Z}_{2}(G(p))$. The free action of $\mathbb{Z}_{2}^{n}$ on $P \times \mathbb{Z}_{2}^{n}$ descends to an action on $M(\lambda)$ with quotient $P$. It is easy to show that the action is locally standard. On the other hand, Davis and Januszkiewicz introduced the notion of what is called a small cover in [3]. A small cover is a smooth closed manifold $M^{n}$ with a locally standard $\mathbb{Z}_{2}^{n}$-action such that its orbit space is a simple convex polytope. Thus $M(\lambda)$ is a small cover over $P$.

Two small covers $M_{1}$ and $M_{2}$ are said to be weakly $\mathbb{Z}_{2}^{n}$-equivariantly homeomorphic (or simply weakly $\mathbb{Z}_{2}^{n}$-homeomorphic) if there is an automorphism $\varphi: \mathbb{Z}_{2}^{n} \rightarrow \mathbb{Z}_{2}^{n}$ and a homeomorphism $f: M_{1} \rightarrow M_{2}$ such that $f(t \cdot x)=\varphi(t) \cdot f(x)$ for every $t \in \mathbb{Z}_{2}^{n}$ and $x \in M_{1}$. If $\varphi$ is the identity, then $M_{1}$ and $M_{2}$ are $\mathbb{Z}_{2}^{n}$-homeomorphic. Following Davis and Januszkiewicz, two small covers $M_{1}$ and $M_{2}$ over $P$ are said 
to be Davis-Januszkiewicz equivalent (or simply, $D-J$ equivalent) if there is a weakly $\mathbb{Z}_{2}^{n}$-homeomorphism $f: M_{1} \rightarrow M_{2}$ covering the identity on $P$.

Let $\operatorname{cf}(P)$ denote the set of all characteristic functions over $P$.

Theorem 1.1 [3] All small covers over $P$ are given by $\{M(\lambda) \mid \lambda \in \operatorname{cf}(P)\}$, ie, for each small cover $M$ over $P$, there is a characteristic function $\lambda$ with $\mathbb{Z}_{2}^{n}-$ homeomorphism $M(\lambda) \rightarrow M$ covering the identity on $P$.

There is a natural free left action of $\operatorname{GL}\left(n, \mathbb{Z}_{2}\right)$ on $\operatorname{cf}(P)$ defined by the correspondence $\lambda \mapsto \sigma \circ \lambda$. This action induces D-J equivalence on $\operatorname{cf}(P)$. Hence the number of D-J equivalence classes over $P$ is $\left|\mathrm{GL}\left(n, \mathbb{Z}_{2}\right) \backslash \operatorname{cf}(P)\right|$. In recent years, numerous studies have attempted to enumerate the number of equivalence classes of all small covers over a specific polytope. In [5], Garrison and Scott used a computer program to show that the number of D-J classes over a dodecahedron is 2165. Moreover they calculated that the number of homeomorphism classes of all small covers over a dodecahedron is 25. In [1], Cai, Chen and Lü calculated the number of D-J classes and $\mathbb{Z}_{2}^{3}$-equivariant homeomorphism classes over 3 -dimensional prisms.

The arrangement of this paper is as follows. In Section 2 we study small covers over cubes, prove some algebraic lemmas and use a combinatorial method to prove that the number of small covers over an $n$-cube up to D-J equivalence is equal to the number of acyclic digraphs with $n$ labeled nodes. Moreover we give the formula of the number of small covers over a product of simplices up to D-J equivalence. In Section 3, we obtain a calculation formula of the number of equivariant homeomorphism classes of all small covers over cubes. In Section 4, we show that the number of weakly homeomorphism classes is less than or equal to the number of acyclic digraphs with $n$ unlabeled nodes.

\section{Small covers over cubes and acyclic digraphs}

\subsection{Small covers over an $n$-cube}

Recall that we may assign an $(n \times m)$-matrix $\Lambda$ to an element $\lambda \in \operatorname{cf}(P)$ by ordering the facets and choosing a basis for $\left(\mathbb{Z}_{2}\right)^{n}$. Let

$$
\Lambda=\left(\lambda\left(F_{1}\right) \cdots \lambda\left(F_{m}\right)\right)=(A \mid B),
$$

where $A$ is an $n \times n$ matrix and $B$ is an $n \times(m-n)$ matrix. Since there is a 1-1 correspondence between the D-J classes over $P$ and $\operatorname{GL}\left(n, \mathbb{Z}_{2}\right) \backslash \operatorname{cf}(P)$, the refined representative in its coset class is given by $\Lambda \sim\left(E_{n} \mid A^{-1} B\right)$, where $E_{n}$ is an identity matrix of size $n$. Denote $\Lambda_{*}=A^{-1} B$. We refer to $\Lambda$ as the refined form of characteristic function $\lambda$ and call $\Lambda_{*}$ its reduced submatrix. 
When $P$ is an $n$-dimensional cube $I^{n}$, since the number of facets of $I^{n}$ is $2 n, \Lambda$ is an $n \times 2 n$ matrix, ie, $\Lambda_{*}$ is an $n \times n$ matrix. We shall additionally assume that the facets $F_{j}$ and $F_{n+j}$ do not intersect for $1 \leq j \leq n$. Then the nonsingularity condition of $\lambda$ is equivalent to the following: every principal minor of $\Lambda_{*}$ is 1 . Let $M(n)$ be the set of $\mathbb{Z}_{2}$-matrices of size $n$ all of whose principal minors are 1 . Then we have the following 1-1 correspondence:

$$
\left\{\mathrm{D}-\mathrm{J} \text { classes over } I^{n}\right\} \leftrightarrow M(n) .
$$

Given a permutation $\mu$ of $n$ elements, denote by $P(\mu)$ the corresponding $n \times n$ permutation matrix, which has units in positions $(\mu(i), i)$ for $1 \leq i \leq n$, and zeros otherwise. There is the symmetric group action on $n \times n$ matrices by conjugations $A \mapsto P(\mu)^{-1} A P(\mu)$. Now we give the technical lemma which first appeared in Dobrinskaya [4]. But we cite it from Masuda and Panov [7, Lemma 3.3].

Lemma 2.1 [7] Let $R$ be a commutative integral domain with an identity element 1, and let $A$ be an $n \times n$ matrix with entries in $R$. Suppose that every proper principal minor of $A$ is 1 . If $\operatorname{det} A=1$, then $A$ is conjugate to a unipotent upper triangular matrix by a permutation matrix, and otherwise to a matrix of the form

$$
\left(\begin{array}{ccccc}
1 & b_{1} & 0 & \cdots & 0 \\
0 & 1 & b_{2} & \cdots & 0 \\
\vdots & \vdots & \ddots & \ddots & \vdots \\
0 & 0 & \cdots & 1 & b_{n-1} \\
b_{n} & 0 & \cdots & 0 & 1
\end{array}\right)
$$

where $b_{i} \neq 0$ for every $i$.

Theorem 2.2 The number of acyclic digraphs with $n$ labeled nodes is equal to the number of $D-J$ equivalence classes of all small covers over $I^{n}$.

A "digraph" means a graph with at most one edge directed from a vertex $i$ to a vertex $j$, for $1 \leq i \leq n, 1 \leq j \leq n$. An "acyclic" means that there is no cycle of any length.

Proof Let $G$ be a digraph with $n$ labeled nodes. Let $A(G)$ be the vertex adjacency matrix of $G$ with $\mathbb{Z}_{2}$ entries. Set $B(G):=E_{n}+A(G)$. Note that a conjugation action at $B(G)$ can be regarded as relabelling nodes. It is obvious that $G$ is acyclic if and only if $A(G)$ would be a strictly upper triangular matrix by conjugation, ie, $B(G)$ would be an upper triangular matrix with diagonal entries 1 . Note that the determinant of $B(G)$ is 1 . Let $\mathcal{G}_{n}$ be the set of acyclic digraphs with labeled $n$ nodes. Define $\phi: \mathcal{G}_{n} \rightarrow M(n)$ by $G \mapsto B(G)$. We claim that it is indeed a bijection between $\mathcal{G}_{n}$ and $M(n)$. Let $G$ 
be an acyclic digraph with $n$ labeled nodes and let $V$ be the node set of $G$. For any subset $V^{\prime}$ of $V$, consider the induced subgraph $G^{\prime}$ by $V^{\prime}$. Then it is also an acyclic digraph. If $\left|V^{\prime}\right|=k$, then $B\left(G^{\prime}\right)$ is a $k$-rowed submatrix of $B(G)$. (A $k$-rowed submatrix of $n \times n$ matrix $B$ is a $k \times k$ submatrix of $B$ whose entries $b_{i, j}$ have indices $i$ and $j$ that are the elements of the same $k$-element subset of $\{1, \ldots, n\}$.) Thus the determinant of $B\left(G^{\prime}\right)$ is the principal minor of $B(G)$. Since det $B\left(G^{\prime}\right)=1$ for any acyclic digraph $G^{\prime}$, hence $B(G) \in M(n)$. Thus $\phi$ is well-defined. And it is injective since $G \mapsto A(G)$ is injective. Let $B$ be an element of $M(n)$. Note that every diagonal entry of $B$ is 1 . Then there is a digraph $G$ such that $B(G)=B$. By Lemma 2.1, since every principal minor of $B$ is $1, B$ is conjugated to a unipotent upper triangular matrix. This implies $G$ is acyclic, so $\phi$ is surjective.

Acyclic digraphs were counted by Robinson in [9] and by Stanley in [11].

Theorem $2.3 \quad[9 ; 11]$ Let $R_{n}$ be the number of acyclic digraphs with $n$ labeled nodes. Then

$$
R_{n}=\sum_{k=1}^{n}(-1)^{k+1}\left(\begin{array}{l}
n \\
k
\end{array}\right) 2^{k(n-k)} R_{n-k} .
$$

Here are a few values of $R_{n}$ :

\begin{tabular}{|c|ccccccccc}
\hline$n$ & 0 & 1 & 2 & 3 & 4 & 5 & 6 & 7 & $\cdots$ \\
\hline$R_{n}$ & 1 & 1 & 3 & 25 & 543 & 29281 & 3781503 & 1138779265 & $\cdots$ \\
\hline
\end{tabular}

Now consider $A \in M(n)$. Note that $A$ is a $\mathbb{Z}_{2}$-matrix. One may regard $A$ as a real matrix with entries in $\{0,1\}$. We simply call it a $(0,1)$-matrix. Then every principal minor of $A$ is an odd number.

Lemma 2.4 Let $A$ be a $(0,1)$-matrix all of whose principal minors are odd. Then every principal minor of $A$ is 1 .

Proof We shall use an induction on $n$. When $n=1$, it is obvious. Assume that it holds for all matrices of size $\leq n-1$. By the induction hypothesis, every proper principal minor of $A$ is +1 . If $\operatorname{det}(A) \neq 1$, then $\operatorname{det}(A)=1 \pm \prod b_{i}$ by Lemma 2.1 . However $A$ is a $(0,1)$-matrix and a conjugation action is a permutation of the rows and columns of $A$, and hence $b_{i}$ 's must be 1 . Thus the determinant of $A$ is even. This is a contradiction. Thus $\operatorname{det}(A)=1$.

Corollary 2.5 The number of acyclic digraphs with $n$ labeled nodes is equal to the number of real $(0,1)$-matrices all of whose principal minors are 1.

Proof This follows immediately from Lemma 2.4 and Theorem 2.2. 
Remark 2.6 We know that the number of acyclic digraphs with $n$ labeled nodes is equal to the number of $(0,1)$-matrices whose eigenvalues are positive. It was conjectured by Weisstein in 2001 and proved by McKay et al [8]. Thus it can be easily checked that all eigenvalues of a $(0,1)$-matrix are positive if and only if all of its principal minors are 1.

Remark 2.7 We can define a quasitoric manifold with $\left(S^{1}\right)^{n}$-action as we did above. In this case, the reduced submatrix $\Lambda_{*}$ of a characteristic function is an integer matrix. When $P$ is an $n$-cube, $\Lambda_{*}$ is an $n \times n$ matrix all of whose principal minors are \pm 1 . Especially, if every principal minor of $-\Lambda_{*}$ is 1 , then the quasitoric manifold is equivalent to a Bott tower. We refer the reader to Masuda and Panov [7]. By Lemma 2.4, for a small cover over $I^{n}$ with $\Lambda_{*}$, there is the Bott tower over $I^{n}$ such that the characteristic function whose reduced submatrix is $-\Lambda_{*}$ as a $(0,1)-$ matrix.

\subsection{Small covers over a product of simplices}

The above processes can be extended to the case of a product of simplices. Let $P=\prod_{i=1}^{l} \Delta^{n_{i}}$ with $\sum_{i=1}^{l} n_{i}=n$, where $\Delta^{n_{i}}$ is the $n_{i}$-simplex for $i=1, \ldots, l$. Let $\left\{f_{0}^{i}, \ldots, f_{n_{i}}^{i}\right\}$ be the set of facets of the simplex $\Delta^{n_{i}}$. Thus the set of facets of $P$ is

$$
\left\{F_{k_{i}}^{i} \mid 0 \leq k_{i} \leq n_{i}, i=1, \ldots, l\right\}
$$

where $F_{k_{i}}^{i}=\Delta^{n_{1}} \times \cdots \times \Delta^{n_{i-1}} \times f_{k_{i}}^{i} \times \Delta^{n_{i+1}} \times \cdots \times \Delta^{n_{l}}$. Thus there are $n+l$ facets in $P$. Then the reduced submatrix $\Lambda_{*}$ of a characteristic function over $P$ is an $n \times l$ matrix. On the other hand, $\Lambda_{*}$ can be viewed as an $l \times l$ matrix $\left(\mathbf{v}_{i, j}\right)$ whose entries in the $j$-th row are vectors in $\mathbb{Z}_{2}^{n_{j}}$. We shall call it a vector matrix. We refer the reader to Choi, Masuda and Suh [2] for details. Let $\Lambda_{k_{1} \cdots k_{l}}$ be the $l \times l$ submatrix of $\Lambda$ whose $j$-th row is the $k_{j}$-th row of the $\mathbf{v}_{i, j}$. Then the nonsingularity condition for $\Lambda$ is equivalent that every principal minor of $\Lambda_{k_{1} \cdots k_{l}}$ is 1 for any $1 \leq k_{1} \leq n_{1}, \ldots, 1 \leq k_{l} \leq n_{l}$.

Theorem 2.8 Let $\sharp \mathrm{DJ}\left(\prod_{i=1}^{l} \Delta^{n_{i}}\right)$ denote the number of $D-J$ equivalence classes over $\prod_{i=1}^{l} \Delta^{n_{i}}$. Then

$$
\sharp \mathrm{DJ}\left(\prod_{i=1}^{l} \Delta^{n_{i}}\right)=\sum_{G \in \mathcal{G}_{l}} \prod_{v_{i} \in V(G)}\left(2^{n_{i}}-1\right)^{\mathrm{outdeg}\left(v_{i}\right)},
$$

where $\mathcal{G}_{l}$ is the set of acyclic digraphs with labeled $l$ nodes and $V(G)=\left\{v_{1}, \ldots, v_{l}\right\}$ is the labeled vertex set of $G$. 
Proof Let $\Lambda_{*}=\left(\mathbf{v}_{i, j}\right)$ be the reduced submatrix of a characteristic function over $P$ with $\mathbf{v}_{i, j} \in \mathbb{Z}_{2}^{n_{i}}$. Denote by $B\left(\Lambda_{*}\right):=\left(b_{i, j}\right)$ the corresponding $l \times l$ matrix over $\mathbb{Z}_{2}$, which has units in positions $(i, j)$ if $\mathbf{v}_{i, j}$ is nonzero, and zeros otherwise. Define the map $\psi$ from $\left\{\mathrm{GL}\left(n, \mathbb{Z}_{2}\right) \backslash \operatorname{cf}(P)\right\}$ to $\mathcal{G}_{l}$ by $\Lambda_{*} \mapsto G$ such that the adjacency matrix of $G$ is $B\left(\Lambda_{*}\right)-E_{l}$. By using similar arguments of the proof of Theorem 2.2, we can prove that $\psi$ is well-defined. Thus the number of characteristic functions is $\sum_{G \in \mathcal{G}_{l}}\left|\psi^{-1}(G)\right|$. Let $G$ be an element of $\mathcal{G}_{l}$ and $\Lambda_{*}=\left(\mathbf{v}_{i, j}\right)$ be an $l \times l$ vector matrix such with $\psi\left(\Lambda_{*}\right)=G$. Note that an directed edge from $i$ to $j$ in $G$ is associated to a nonzero $\mathbf{v}_{i, j}$ and $\mathbf{v}_{i, j} \in \mathbb{Z}_{2}^{n_{i}}$. Note that $B\left(\Lambda_{*}\right)$ is conjugated to a unipotent upper triangular matrix. Therefore the nonsingularity condition holds for arbitrary nonzero vectors in nondiagonal entries, ie, we have $2^{n_{i}}-1$ choices for each nonzero vector $\mathbf{v}_{i, j}$. Thus $\left|\psi^{-1}(G)\right|=\prod_{e \in E(G)}\left(2^{n_{i}(e)}-1\right)=\prod_{v_{i} \in V(G)}\left(2^{n_{i}}-1\right)^{\text {outdeg }\left(v_{i}\right)}$, where $E(G)$ is the set of directed edges of $G$ and $i(e)$ is the label of the initial vertex of $e \in E(G)$.

Example 2.9 $l=2: \sharp \mathrm{DJ}\left(\Delta^{n_{1}} \times \Delta^{n_{2}}\right)=1+\left(2^{n_{1}}-1\right)+\left(2^{n_{2}}-1\right)$.

$l=3$ : Letting $x_{i}=2^{n_{i}}-1$ for $i=1,2,3$,

$$
\begin{aligned}
& \sharp \mathrm{DJ}\left(\Delta^{n_{1}} \times \Delta^{n_{2}} \times \Delta^{n_{3}}\right) \\
& =1+2\left(x_{1}+x_{2}+x_{3}\right)+\left(x_{1}+x_{2}+x_{3}\right)^{2}+\left(x_{1} x_{2}+x_{2} x_{3}+x_{3} x_{1}\right) \\
& +\left(x_{1}+x_{2}+x_{3}\right)\left(x_{1}^{2}+x_{2}^{2}+x_{3}^{2}\right)-x_{1}^{3}-x_{2}^{3}-x_{3}^{3} \text {. }
\end{aligned}
$$

\section{Counting $\mathbb{Z}_{2}^{n}$-equivariant homeomorphism classes}

Let $P$ be a simple convex polytope of dimension $n$ and let $\mathcal{F}(P)$ be the set of faces of $P$. An automorphism of $\mathcal{F}(P)$ is a bijection from $\mathcal{F}(P)$ to itself which preserves the poset structure of all faces of $P$. Let $\operatorname{Aut}(\mathcal{F}(P))$ denote the group of automorphisms of $\mathcal{F}(P)$. One can define the right action of $\operatorname{Aut}(\mathcal{F}(P))$ on $\operatorname{cf}(P)$ by $\lambda \times h \mapsto \lambda \circ h$, where $\lambda \in \operatorname{cf}(P)$ and $h \in \operatorname{Aut}(\mathcal{F}(P))$. The following theorem is well-known. We refer the reader to Lü and Masuda [6].

Theorem 3.1 Two small covers over an $n$-dimensional simple convex polytope $P$ are $\mathbb{Z}_{2}^{n}$-equivariantly homeomorphic if and only if there is an element $h \in \operatorname{Aut}(\mathcal{F}(P)$ ) such that $\lambda_{1}=\lambda_{2} \circ h$, where $\lambda_{1}$ and $\lambda_{2}$ are characteristic functions of small covers.

Thus we are going to count the orbits of $\operatorname{cf}\left(I^{n}\right)$ under the action of $\operatorname{Aut}\left(\mathcal{F}\left(I^{n}\right)\right)$. The Burnside's formula is very useful in the enumeration of the number of orbits.

Lemma 3.2 (Burnside's formula) Let $G$ be a finite group acting on a finite set $X$. Then the number of orbits of $X$ under the $G$-action is equal to $\frac{1}{|G|} \sum_{g \in G}\left|X^{g}\right|$, where $X^{g}=\{x \in X \mid g x=x\}$. 
Theorem 3.3 Let $Q_{n}$ be the number of $\mathbb{Z}_{2}^{n}$-equivariant homeomorphism classes of small covers over $I^{n}$ and let $R_{k}$ be the number of acyclic digraphs with $k$ labeled nodes. Then

$$
Q_{n}=\frac{\sum_{k=0}^{n}\left(\begin{array}{l}
n \\
k
\end{array}\right) 2^{k(n-k)} R_{k}}{2^{n} n !} \cdot \prod_{i=0}^{n-1}\left(2^{n}-2^{i}\right) .
$$

Proof All elements of $\operatorname{Aut}\left(\mathcal{F}\left(I^{n}\right)\right)$ can be written in a simple form as follows:

$$
\mu \cdot \chi_{1}^{e_{1}} \cdots \cdots \chi_{n}^{e_{n}}, e_{j} \in \mathbb{Z}_{2}
$$

with a permutation $\mu \in S_{n}$ and reflections $\chi_{1}, \ldots, \chi_{n}$. Hence $\left|\operatorname{Aut}\left(\mathcal{F}\left(I^{n}\right)\right)\right|=2^{n} n !$. Note that $\mu$ is a permutation of the pairs of opposite facets and $\chi_{i}$ is the interchange of $i$-th opposite facets for each $i$. For some $g=\mu \chi_{1}^{e_{1}} \cdots \chi_{n}^{e_{n}} \in \operatorname{Aut}\left(\mathcal{F}\left(I^{n}\right)\right)$, let $\operatorname{cf}\left(I^{n}\right)^{g}$ denote the set of elements in $\operatorname{cf}\left(I^{n}\right)$ fixed by $g$. First, we claim that if $\operatorname{cf}\left(I^{n}\right)^{g}$ is nonempty, then $\mu=1$. Let $\lambda \in \operatorname{cf}\left(I^{n}\right)^{g}$ and $\mathcal{F}\left(I^{n}\right)=\left\{F_{1}, \ldots, F_{2 n}\right\}$ be the set of facets of $I^{n}$ such that $F_{i} \cap F_{n+i}=\varnothing$ for all $i=1, \ldots, n$. Note that the nonsingularity condition implies the determinant of $\left(\lambda\left(F_{\epsilon(1)}\right) \cdots \lambda\left(F_{\epsilon(n)}\right)\right)$ is 1 , where $\epsilon(t)$ is either $t$ or $n+t$. Thus there is no pair $F_{i}, F_{j}$ such that $\lambda\left(F_{i}\right)=\lambda\left(F_{j}\right)$ and $n \nmid i-j$. This shows that $\mu=1$. Now, we are going to enumerate $\left|\operatorname{cf}\left(I^{n}\right)^{g}\right|$ when $\mu=1$. We may assume $g=\chi_{1} \cdots \chi_{k}$ for some $k$. Let $\lambda$ be an element of $\operatorname{cf}\left(I^{n}\right)^{g}$ and let $\Lambda$ be an $n \times 2 n$ matrix corresponding to $\lambda$. Note that $\Lambda=(A \mid B)=A \cdot\left(E_{n} \mid \Lambda_{*}\right)$, where $\Lambda_{*}=A^{-1} B$. Note that $\lambda$ is fixed by $g$ if and only if the first $k$ columns of $A$ and $B$ are the same. Thus $\Lambda_{*}$ is of the following form:

$$
\left(\begin{array}{cc}
E_{k} & S \\
0 & T
\end{array}\right)
$$

where $E_{k}$ is an identity matrix of size $k, T$ is an $(n-k) \times(n-k)$ matrix and $S$ is a $k \times(n-k)$ matrix. Note that $\Lambda_{*} \in M(n)$ if and only if $T \in M(k)$. This implies $\left|\operatorname{cf}\left(I^{n}\right)^{g}\right|=\left|\operatorname{GL}\left(n, \mathbb{Z}_{2}\right)\right| \times 2^{k(n-k)} R_{k}$. Note that $\left|\operatorname{cf}\left(I^{n}\right)^{g}\right|$ is independent of choices of $k \chi_{i}$ 's. Thus by Burnside's formula,

$$
Q_{n}=\frac{\sum_{k=0}^{n}\left(\begin{array}{l}
n \\
k
\end{array}\right) 2^{k(n-k)} R_{k}}{\left|\operatorname{Aut}\left(I^{n}\right)\right|} \cdot\left|\operatorname{GL}\left(n, \mathbb{Z}_{2}\right)\right| .
$$

The theorem is proved with the well-known fact $\left|\operatorname{GL}\left(n, \mathbb{Z}_{2}\right)\right|=\prod_{i=0}^{n-1}\left(2^{n}-2^{i}\right)$.

Here are a few values of $\mathcal{Q}_{n}$ :

\begin{tabular}{|c|ccccccc}
\hline$n$ & 0 & 1 & 2 & 3 & 4 & 5 & $\cdots$ \\
\hline$Q_{n}$ & 1 & 1 & 6 & 259 & 87360 & 236240088 & $\cdots$ \\
\hline
\end{tabular}




\section{Upper bounds of the numbers of homeomorphism classes}

By Theorem 3.1, we have a one-to-one correspondence between the set of weakly equivariant homeomorphism classes of small covers over simple polytope $P$ and the double coset class by $\operatorname{GL}\left(n, \mathbb{Z}_{2}\right)$ and $\operatorname{Aut}(\mathcal{F}(P))$ on $\operatorname{cf}(P)$. Let $T_{n}$ be the number of weakly $\mathbb{Z}_{2}^{n}$-equivariant homeomorphism classes of small covers over $I^{n}$. Then

$$
T_{n}=\left|\operatorname{GL}\left(n, \mathbb{Z}_{2}\right) \backslash \operatorname{cf}\left(I^{n}\right) / \operatorname{Aut}\left(\mathcal{F}\left(I^{n}\right)\right)\right|=\left|M(n) / \operatorname{Aut}\left(\mathcal{F}\left(I^{n}\right)\right)\right|
$$

where $M(n)$ is the set of $\mathbb{Z}_{2}$-matrices of size $n$ all of whose principal minors are 1 . Recall that $\operatorname{Aut}\left(\mathcal{F}\left(I^{n}\right)\right)$ consists of elements of the form $\mu \cdot \chi_{1}^{e_{1}} \cdots \cdots \chi_{n}^{e_{n}}, e_{j} \in \mathbb{Z}_{2}$ with a permutation $\mu \in S_{n}$ and reflections $\chi_{1}=\cdots=\chi_{n}$. Consider the permutation group $S_{n}=\left\{g \in \operatorname{Aut}\left(\mathcal{F}\left(I^{n}\right)\right) \mid g=\mu \chi_{1}^{0} \cdots \chi_{n}^{0}\right\}$ as a subgroup of $\operatorname{Aut}\left(\mathcal{F}\left(I^{n}\right)\right)$. Then $S_{n}$ acts on the set of facets of $I^{n}$ by permutating the pairs of opposite facets. Let $\Lambda$ be an $n \times 2 n$ characteristic matrix. Then $\mu \in S_{n}$ acts as

$$
\Lambda \mapsto \Lambda \cdot\left(\begin{array}{cc}
P(\mu) & 0 \\
0 & P(\mu)
\end{array}\right) .
$$

Thus we have $\left(E_{n} \mid \Lambda_{*}\right) \mapsto\left(P(\mu) \mid \Lambda_{*} P(\mu)\right) \sim\left(E_{n} \mid P(\mu)^{-1} \Lambda_{*} P(\mu)\right)$. This implies that the action of $S_{n}$ on $M(n)$ is the conjugation action. That action is a relabeling on nodes of acyclic digraphs. Hence we have the following theorem and corollary:

Theorem 4.1 The number of weakly $\mathbb{Z}_{2}^{n}$-equivariant homeomorphism classes of small covers over $I^{n}$ is less than or equal to the number of acyclic digraphs with $n$ unlabeled nodes.

Corollary 4.2 The number of homeomorphism classes of small covers over $I^{n}$ is less than or equal to the number of acyclic digraphs with $n$ unlabeled nodes.

Acyclic digraphs with unlabeled nodes were counted by Robinson in [10].

Here are a few values of $T_{n}$ :

\begin{tabular}{|c|ccccccccc}
\hline$n$ & 0 & 1 & 2 & 3 & 4 & 5 & 6 & 7 & $\cdots$ \\
\hline$T_{n}$ & 1 & 1 & 2 & 6 & 31 & 302 & 5984 & 243668 & $\cdots$ \\
\hline
\end{tabular}

Acknowledgements This work was partially supported by the SRC Program of Korea Science and Engineering Foundation (KOSEF) grant funded by the Korea government (MOST) (No. R11-2007-035-02002-0). The author is grateful to his advisor, Professor Dong Youp Suh, for his encouragement and a number of comments and suggestions. Also the author thanks Professor Mikiya Masuda for his many useful suggestions and Jang Soo Kim for his comments, which improved the proof of Theorem 2.2. 


\section{References}

[1] M Cai, X Chen, Z Lü, Small covers over prisms, Topology Appl. 154 (2007) 22282234 MR2328006

[2] S Choi, M Masuda, D Y Suh, Quasitoric manifolds over a product of simplices, to appear in Osaka J. Math. arXiv:0803.2749

[3] M W Davis, T Januszkiewicz, Convex polytopes, Coxeter orbifolds and torus actions, Duke Math. J. 62 (1991) 417-451 MR1104531

[4] N È Dobrinskaya, The classification problem for quasitoric manifolds over a given polytope, Funktsional. Anal. i Prilozhen. 35 (2001) 3-11 MR1850429

[5] A Garrison, R Scott, Small covers of the dodecahedron and the 120-cell, Proc. Amer. Math. Soc. 131 (2003) 963-971 MR1937435

[6] Z Lü, M Masuda, Equivariant classification of 2-torus manifolds, Preprint (2008)

[7] M Masuda, T E Panov, Semifree circle actions, Bott towers, and quasitoric manifolds, Sbornik Math. 199 (2008) 1201-1223

[8] B D McKay, F E Oggier, G F Royle, N A Sloane, I M Wanless, HS Wilf, Acyclic digraphs and eigenvalues of (0,1)-matrices, J. Integer Seq. 7 (2004) Article 04.3.3, 5 pp. MR2085343

[9] R W Robinson, Enumeration of acyclic digraphs, from: "Proc. Second Chapel Hill Conf. on Combinatorial Mathematics and its Applications", Univ. North Carolina, Chapel Hill (1970) 391-399 MR0276143

[10] R W Robinson, Counting unlabeled acyclic digraphs, from: "Combinatorial mathematics, V (Proc. Fifth Austral. Conf., Roy. Melbourne Inst. Tech., 1976)”, (C H C Little, editor), Lecture Notes in Math. 622, Springer, Berlin (1977) 28-43 MR0476569

[11] RP Stanley, Acyclic orientations of graphs, Discrete Math. 5 (1973) 171-178 MR0317988

KAIST, Department of Mathematical Sciences

335 Gwahangno, Yuseong-gu, Daejeon, 305-701, South Korea

choisy@kaist.ac. kr

http://topology.kaist.ac.kr/schoi

Received: 3 October 2008 Revised: 4 November 2008 